\title{
Embedded Digital Signal Processing for Digital Ultrasound Imaging
}

\author{
Mawia A. Hassan $^{1,2}$, Abou-Bakr M. Youssef ${ }^{1}$, Yasser M. Kadah ${ }^{1}$ \\ ${ }^{1}$ System \& Biomedical Engineering Department, Cairo University, Giza, Egypt, Biomedical Engineering \\ Department, Sudan University of Science \& Technology, Khartoum, Sudan \\ E-mail:mah@k-space.org
}

\begin{abstract}
Ultrasound imaging is an efficient, noninvasive, method for medical diagnosis. Efficient implementations of digital ultrasound systems on embedded digital signal processing on FPGA, this miniaturization enables a design with low power consumption, low noise, and light weight. This paper proposed embedded digital signal processing (DSP) for digital ultrasound imaging on FPGA (Xilinx, Inc.). The DSP was composed of FIR Hilbert transform filter, which was used to generate Quadrature component (Q) from the In-phase component (I) of the ultrasound data. The envelope (magnitude) of the received echo was computed. The implementation has been done in the Virtex-5 FPGA. The objective of this work is to build embedded DSP for ultrasound imaging system using the FIR Hilbert transform filter, which will be described in the methods. The system was consisted of: the pipeline adder block to reconstruct the focus ultrasound line, the bit modifier block to modify the bit of the signal to 16 bit, the FIR Hilbert filter block to obtain the quadrature components, the fractional delay filter (inphase filter) to compensate the delay when we were used a high FIR order, and the envelope detection block to compute the envelope of the in-phase and quadrature components. The Hilbert filter is implemented in the form whereby the zero tap coefficients are not computed and therefore an order L filter uses only L/2 multiplications. This was reducing the computational time by a half. The simulation results of FIR Hilbert filter and the envelope detection are near to the ideal Hilbert. The results of the implementation are good compared to the simulation results. From the implementation result the total estimated power consumption equal to $0.8142 \mathrm{~W}$ and the device utilization was acceptable. It is possible for the system to accept anther devices for further processing. The hardware architecture of the design provided flexibility.
\end{abstract}

Keywords: Embedded DSP; Digital Ultrasound; FIR Hilbert transform filter; FPGA.

\section{INTRODUCTION}

Ultrasound is defined as acoustic waves with frequencies above those which can be detected by the ear, from about $20 \mathrm{KHz}$ to several hundred MHz. Ultrasound for medical applications typically uses only the portion of the ultrasound spectrum from $1 \mathrm{MHz}$ to $50 \mathrm{MHz}$ due to the combined needs of good resolution (small wave length) and good penetrating ability (not too high a frequency) [1].

They are generated by converting a radio frequency (RF) electrical signal into mechanical vibration via a piezoelectric transducer sensor [2]. The ultrasound waves propagate into the tissues of the body where apportion is reflected, which used to generate the ultrasound image. Employed ultrasound waves allow obtaining information about the structure and nature of tissues and organs of the body [3]. It is also used to visualize the heart, and measure the blood flows in arteries and veins [4].

A commonly used approach to image acquisition in ultrasound system is digital beamforming because the analog delay lines impose significant limitations on beamformer performance and more expensive than digital implementations [5]. Digital beamforming, as applied to the medical ultrasound, is defined as phase alignment and summation [2] of signals that are generated from a common source, by received at different times by a multi-elements ultrasound transducer [6].

After delay and sum, the analytic envelope of the signal is calculated as the square root of the sum of the squares of the real and quadrature components [7]. The most accurate way of obtaining the quadrature components was to pass the echo signal through a Hilbert transform [8], because it provides 90-degree phase shift at all frequencies [9]. The envelope then compressed logarithmically to achieve the desired dynamic range for display (8 bits). It is typical to use a log compressor to achieve the desired dynamic range for display. Log transformation compressed the dynamic range with a large variation in pixels values [10].

Different articles introduced the issues involved in digital ultrasound imaging system design including the description of its main components. Embedded digital beamforming was initially done using ApplicationSpecific Integrated Circuits (ASICs) [11]. Many approaches also described the digital signal processing 
algorithms that can be used in digital beamforming signal demodulation [12][13][14]. A compact medical ultrasound beamformer architecture that uses oversampled 1-bit analog-to-digital converters (ADC) is presented in [9]. However, the development in analog-to-digital converter (ADC) and FPGA technologies, make it is possible to use fast analog acquisition like serial low voltage differential signaling (LVDS) interface protocol, which makes the output from an ADC to come as a serial bit stream with drivers on both the ADC and the FPGA to recover the parallel data. This reduces the need for designing the sigma-delta sampling and the signal recovery filters for the oversampled 1-bit data stream while maintaining an optimal.

Our previous work described a modular FPGA-based digital ultrasound beamforming (DBF) [15]. In this paper, we were used the ultrasound line data, which was take from the DBF in [15] to build an embedded DSP for ultrasound imaging system on FPGA (Fig.1). The system composed of: the FIR Hilbert filter block to obtain the quadrature components, the fractional delay filter (in-phase filter) to compensate the delay when we are being used a high FIR order, and the envelope detection block which was computed the envelope of the in-phase and quadrature components. The implemented have been done in the Virtex-5 FPGA [15] [16] [17].

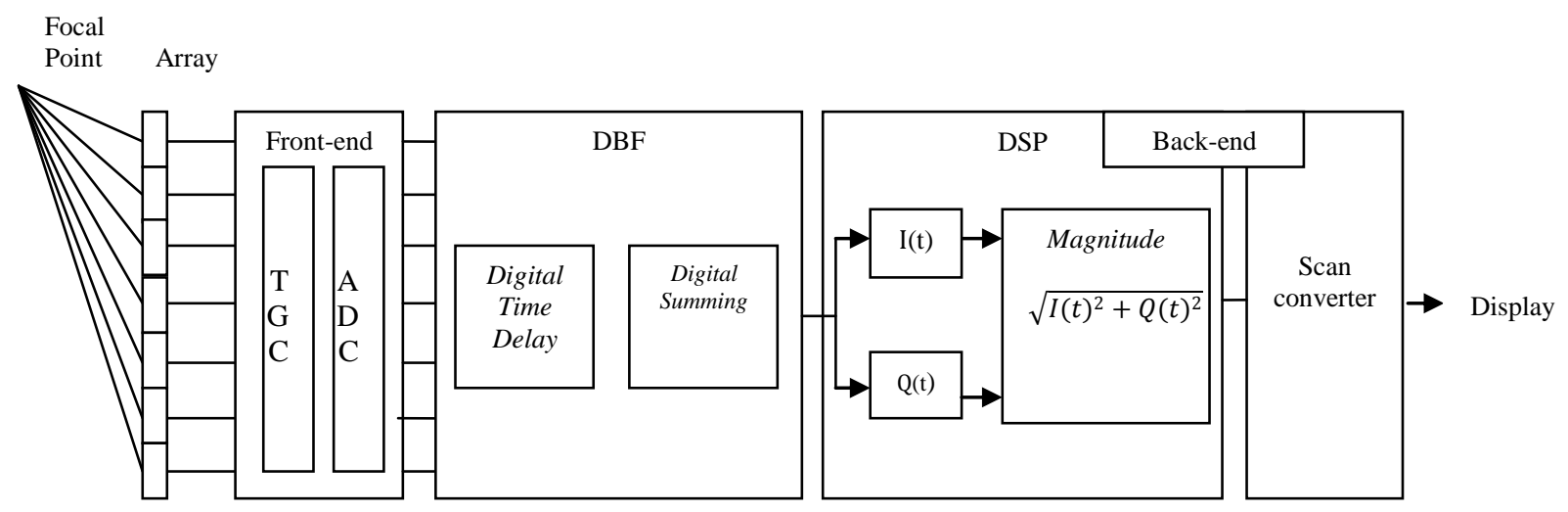

Fig. 1: B-mode processing of the ultrasonic imaging system.

\section{METHODOLOGY}

\section{A. Digital Beamforming (DBF)}

The implemented DBF system (Fig.2) [15] was consisted of: the two 8 channels block and the reconstructed line block. The 8 channel block consist of: the memory block to save the samples data after converted to fixed point type, the delay block implemented by addressable shift register- The delay process is based on sampled delay focusing (SDF) - and the Mcode used to apply the summation of each RF channel samples. The reconstructed block was consisted of the pipelined adder to apply the summation of the two 8 channels blocks output.

\section{B. Hilbert Transform Filter Design}

For linear time invariant (LTI) a finite impulse response (FIR) filter can be described in this form [7]:

$$
y[\mathrm{n}]=b_{0} x[n]+b_{1} x[n-1]+\cdots+b_{M} x[n-M]=\sum_{i=0}^{M} b_{i} x[n-i] .
$$

Where $x$ is the input signal, $y$ is the output signal, and the constants $b_{i}, i=0,1,2, \ldots, \mathrm{M}$, are the coefficients. The designed FIR Hilbert filter can be used to generate the Hilbert transformed data of the received echo signal. The impulse response of the Hilbert filter with length $\mathrm{N}$ (odd number) is defined as [18]:

$$
h[n]=\left\{\begin{array}{c}
\frac{2}{\pi} \frac{\sin ^{2}(\pi(n-\alpha) / 2)}{n-\alpha}, n \neq \alpha, \\
0, n=\alpha
\end{array}\right.
$$




\section{April 26-28, 2011, National Telecommunication Institute, Egypt}

$\alpha=(\mathrm{N}-1) / 2$. We were chosen the filter length equal 9,17,33,65 and 129. The Hamming window was used to reduce the sidelobe effects. The Hilbert filter is implemented in the common optimized form whereby the zero tap coefficients are not computed and therefore an order L filter uses only L/2 multiplications. This was reducing the computational time by a half.

To compensate the delay when using high FIR order, we used fractional delay filter that has the same group delay as the designed FIR filter. A fair approximation of a fractional delay filter is a sampled SINC function with impulse response:

$$
\begin{gathered}
x[n]=(0: \mathrm{N}-1)-\text { D. where } D=(\mathrm{N}-1) / 2 . \\
h=\sin \mathrm{x}[\mathrm{n}] / \mathrm{x}[n] .
\end{gathered}
$$

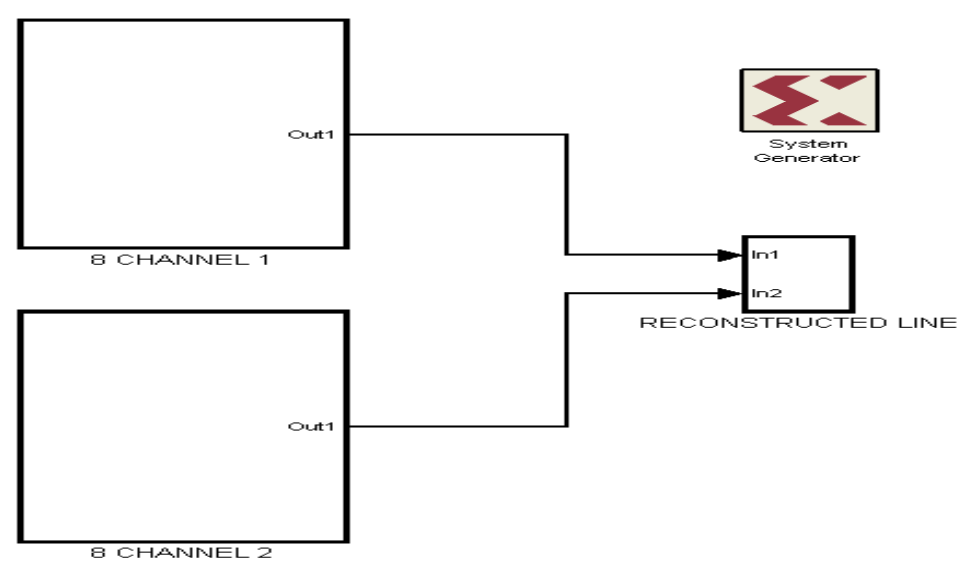

Fig. 2: Architecture implementation of the modular FPGA-Based digital ultrasound receive beamformer blocks [15]

\section{Envelope Detection}

The analytic envelope of the signal was calculated as the square root of the sum of the squares of the real and quadrature components [7] [19] [20]. The most accurate way of obtaining the quadrature components was to pass the echo signal through a Hilbert transform [7], because it provides 90-degree phase shift at all frequencies [9].

We were obtained the quadrature components by design FIR Hilbert filter as we mentioned in 2.2. as a well designed And the frequency response of a 8-, 16-, 32-, 64-, and 128-tap FIR Hilbert filter used in the simulation. Quadrature sampling method, which split a band-pass signal into in-phase and quadrature baseband components [21] as:

$$
\begin{aligned}
& x[t]=\mathrm{A}(\mathrm{t}) \cos \left(\omega_{0} \mathrm{t}+\emptyset(\mathrm{t})\right)=A_{I}(t) \cos \left(\omega_{0} \mathrm{t}\right)+A_{Q}(t) \sin \left(\omega_{0} \mathrm{t}\right) \\
& A(t)=\sqrt{A_{I}^{2}(t)+A_{Q}^{2}(t)} .
\end{aligned}
$$

Where $\omega_{0}$ is the transducer central frequency, $\emptyset$ its phase, $A_{I}, A_{Q}$ is the magnitude of the in-phase and quadrature components and $A(t)$ is the envelope (magnitude) of the received echo at time $t$.

Another method to approximate the Hilbert transform is the obtained by delay the original received echo by $\alpha$ samples, this is depending on the shifting a 90 degree phase from the center frequency of the received echo 


\section{April 26-28, 2011, National Telecommunication Institute, Egypt}

data. This method gave acceptable accuracy when the received signal has a high quality factor Q, otherwise causing a serious error in the detected envelope [22]. Because the ultrasound pulse usually has a relatively low $\mathrm{Q}$, a very fast sampling clock is required to use the time delay method [22].

\section{Implementation Steps}

We were tested a 8-, 16-, 32-, 64-, and 128-tap FIR Hilbert filter, according to the normalized root mean square error (RMSE) between the designed filter and ideal Hilbert transform filter. The values of the RMSE for the five FIR filters are equaled 0.9568, 0.6111, 0.4487, 0.3769, and 0.4166. We were chosen the Hilbert filter with length equal 65 for the implementation because it provided a lower RMSE.

A typical architecture implementation of the FPGA-based for embedded DSP for ultrasound imaging is shown in Figure.1 named the reconstructed line. The implementation was been done using Xilinx system generator (Xilinx, Inc.) and MATLAB simulink (MathWorks, Inc.). The implementation steps are (Fig.3):

1. The summation of the two 8 channels (figure 1) was connected to pipe line adder to reconstructed the focus ultrasound line (table 1).

2. We were modified the bit of the signal to 16 bit using bit modifier block (table 2).

3. The Register block (data presented at the input will appear at the output after one sample period).

4. The FIR Hilbert filter block for applying the quadrature components (table 3).

5. The Fractional delay filter (in-phase filter) to compensate the delay when we are being used a high FIR order (table 4).

6. Then we modified the bit of the signals from step 4 and 5 to 16 bit again using bit modifier blocks. These blocks have the same parameters with step 2 .

7. The Envelope detection block which was computed the envelope of the two signals coming from step 4 and 5 (table 5).

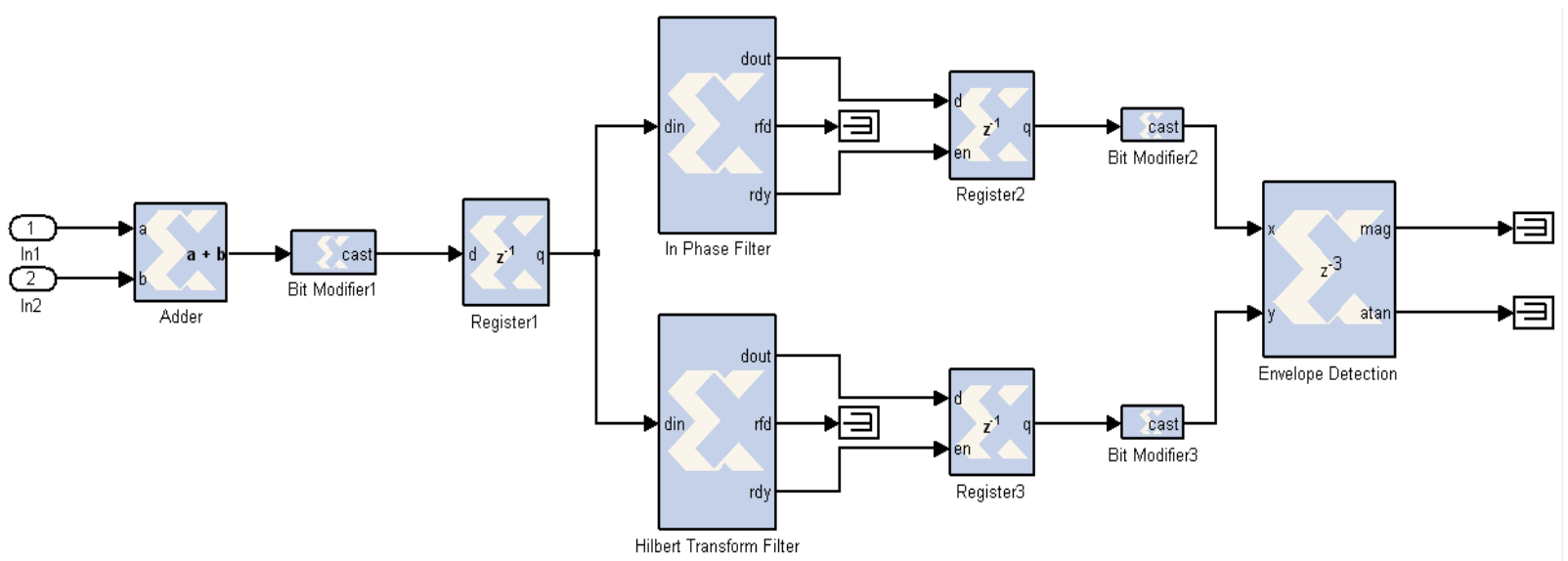

Fig. 3: The inside contents of the implementation blocks

Table 1:The Adder Parameters

\begin{tabular}{|l|l|}
\hline Parameter & Value \\
\hline Operation & Addition \\
\hline Provide carry-in port & off \\
\hline Provide carry-out port & off \\
\hline Provide enable port & off \\
\hline Latency & 0 \\
\hline Precision & Full \\
\hline Output type & Unsigned \\
\hline Number of bits & 16 \\
\hline Binary point & 0 \\
\hline Quantization & Truncate \\
\hline Overflow & Wrap \\
\hline
\end{tabular}

Table 3: The Hilbert filter Parameters

\begin{tabular}{|l|l|}
\hline Parameter & Value \\
\hline Coefficient Vector & y1 \\
\hline Number of Coefficient Sets & 1 \\
\hline Filter Type & Single_Rate \\
\hline Rate Change Type & Integer \\
\hline Interpolation Rate Value & 1 \\
\hline Decimation Rate Value & 1 \\
\hline Zero Pack Factor & 1 \\
\hline Number of Channels & 1 \\
\hline Select format & Hardware_Oversampling_Rate \\
\hline Sample period & 1 \\
\hline Hardware Oversampling Rate & 1 \\
\hline Filter Architecture & Systolic_Multiply_Accumulate \\
\hline
\end{tabular}




\section{April 26-28, 2011, National Telecommunication Institute, Egypt}

Table 2: The Bit Modifier Parameters

\begin{tabular}{|l|l|}
\hline Value & Parameter \\
\hline Signed (2's comp) & Type \\
\hline 16 & Number of bits \\
\hline 0 & Binary point \\
\hline Truncate & Quantization \\
\hline Wrap & Overflow \\
\hline 0 & Latency \\
\hline
\end{tabular}

Table 4: The in-phase filter Parameters

\begin{tabular}{|l|l|}
\hline Parameter & Value \\
\hline Coefficient Vector & y1 \\
\hline Number of Coefficient Sets & 1 \\
\hline Filter Type & Single_Rate \\
\hline Interpolation Rate Value & Integer \\
\hline Decimation Rate Value & 1 \\
\hline Zero Pack Factor & 1 \\
\hline Number of Channels & 1 \\
\hline Select format & 1 \\
\hline Sample period & Hardware_Oversampling_Rate \\
\hline Hardware Oversampling Rate & 1 \\
\hline Filter Architecture & 1 \\
\hline Coefficient Type & Systolic_Multiply_Accumulate \\
\hline Quantization & Signed \\
\hline Coefficient Width & Quantize_Only \\
\hline Coefficient Fractional Bits & 16 \\
\hline Number of Paths & on \\
\hline Output Rounding Mode & 15 \\
\hline Output Width & 33 \\
\hline Optimization Goal & Area \\
\hline Number of samples & 0 \\
\hline
\end{tabular}

Table 5:The envelope detection Parameters

\begin{tabular}{|l|l|}
\hline Parameters & Value \\
\hline $\begin{array}{l}\text { Number of Processing Elements } \\
\text { (integer value starting from 1) }\end{array}$ & 1 \\
\hline X, Y Data Width & 16 \\
\hline X, Y Binary Point Position & 0 \\
\hline $\begin{array}{l}\text { Latency for each Processing } \\
\text { Element }\end{array}$ & {$[1]$} \\
\hline
\end{tabular}

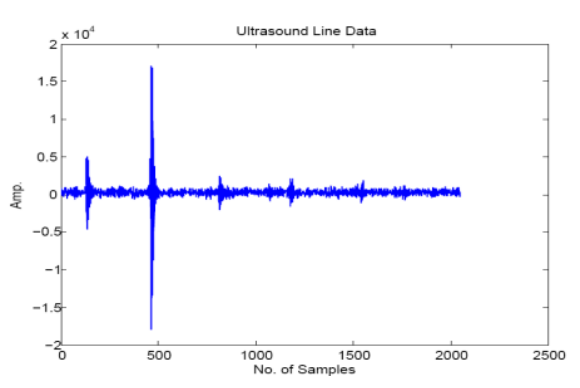

Fig.4: Ultrasound received echo Signal data

\section{RESULTS AND DISCUSSIONS}

\section{A. The Ultrasound data}

We used correct real data obtained from the Biomedical Ultrasound Laboratory, University of Michigan [23]; the data set that was used to generate the results here is under "Acusonl7". The parameters for this data set are as follows: the number of channels was 128 channels, and the ADC sampling rate was 13.8889 MSPS. Linear shape transducer was used to acquire the data with center frequency of $3.5 \mathrm{MHz}$, and element spacing of $0.22 \mathrm{~mm}$. Each ultrasonic A-scan was saved in a record consisted of $2048 \mathrm{RF}$ samples per line each represented in 2 bytes, and the signal averages was 8 . The speed of the ultrasound in the phantom was $1480 \mathrm{~m} / \mathrm{sec}$. The data were acquired for phantom within 6 pins at different positions.

\section{B. Hilbert transform filter design}

Fig. 4 shown the ultrasound received echo taken from the DBF of the phantom within 6 pins. As we mentioned in the methodologies, we used five lengths 8-, 16-, 32-, 64-, and 128-tap FIR Hilbert filter. Fig. $5(\mathrm{a}, \mathrm{b}, \mathrm{c}, \mathrm{d}$, and e) shown the frequency response for each one. Fig. 6 (a) and (b) described the ideal analytical signal and ideal Hilbert frequency spectrum for 70 samples of ultrasound data.

As we mentioned in the methodologies the normalized RMSE between the designed filter and ideal Hilbert transform filter of 64 order FIR Hilbert filter is the smallest one (0.3769), so we use this filter for the simulation and implementation of the ultrasound data. Apply this designed Hilbert filter to the received echo line after delay and sum shown in Fig. 7(a) compared to the ideal Hilbert filter in Fig.7 (b). The RMSE between two signals is being shown in Fig. 7 (c), and the value equal to (0.3769) which mean that the two signals were near the same. Fig.7(d) described the frequency spectrum for ultrasound data. As can be shown the negative frequency was eliminated compared to the ideal Hilbert filter frequency spectrum (Fig 6(b)). When increase the order of the filter would be better (the longer the filter is, the narrower the bandwidth will be). 


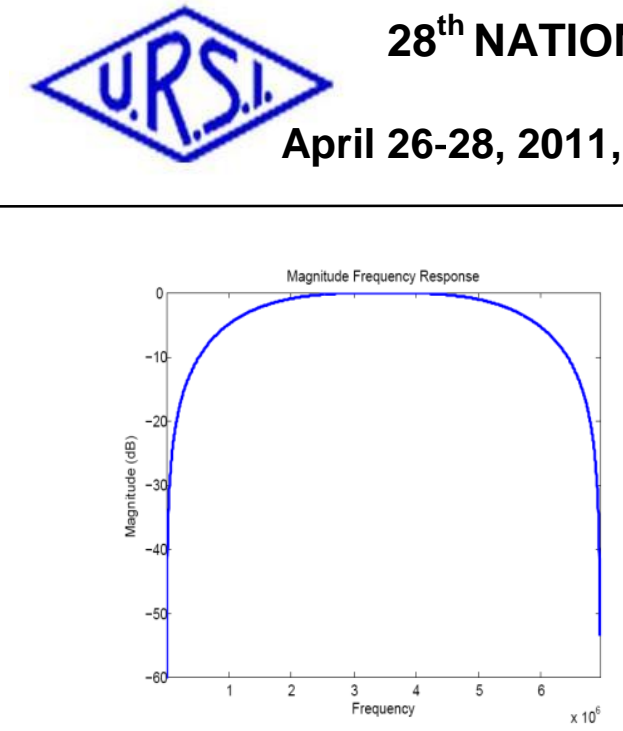

(a)

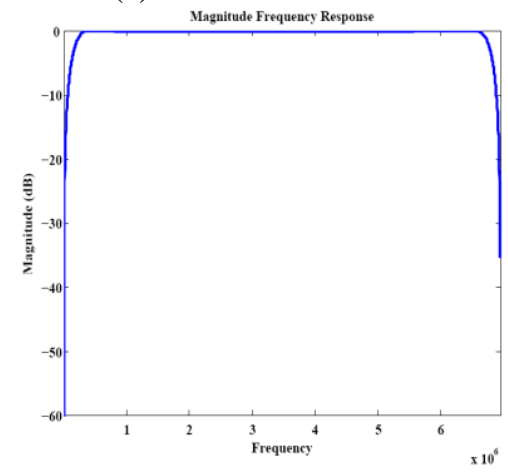

(d)

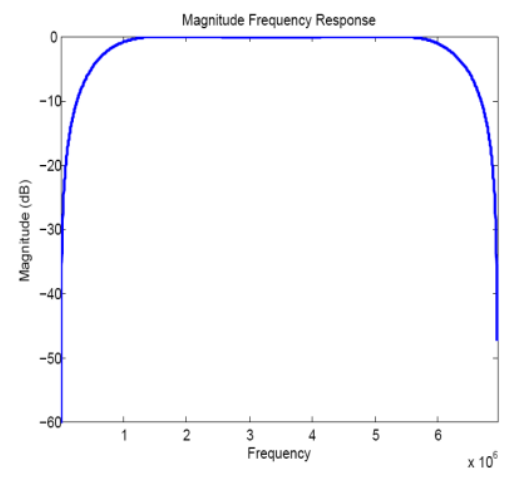

(b)

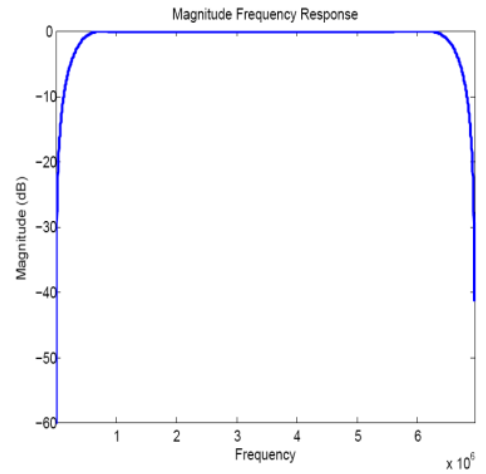

(c)

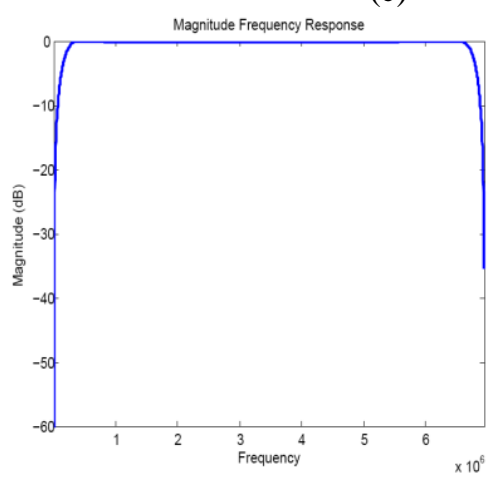

(e)

Fig.5: Magnitude frequency responses for FIR Hilbert filter. (a) 8-tap filter, (b) 16-tap filter, (c) 32-tap filter, (d) 64-tap filter, (e) 128-tap filter.

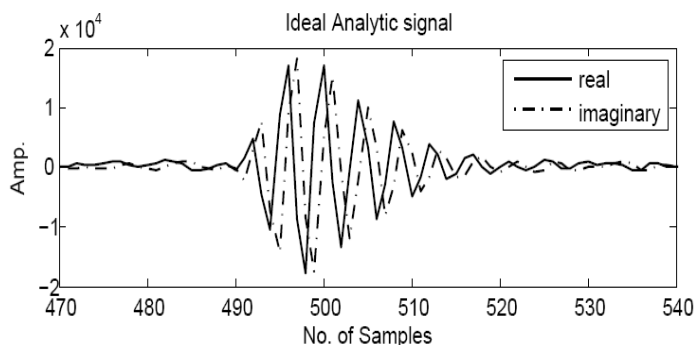

(a)

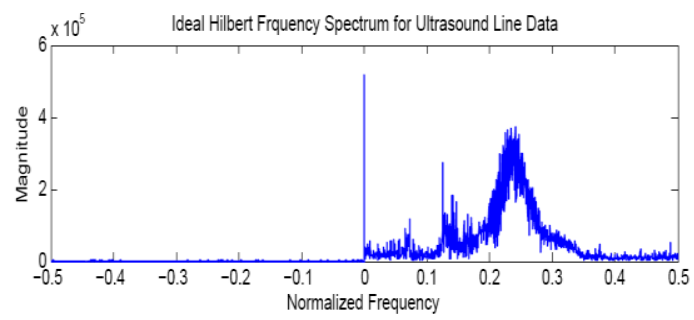

(b)

Fig.6: Ideal Hilbert transform filter. (a) Ideal analytical signal, (b) Ideal Hilbert frequency spectrum.

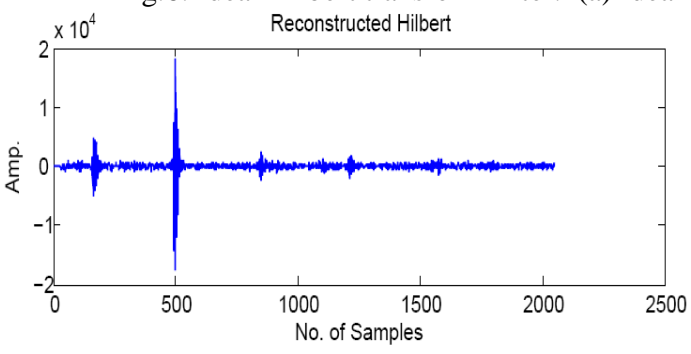

(a)

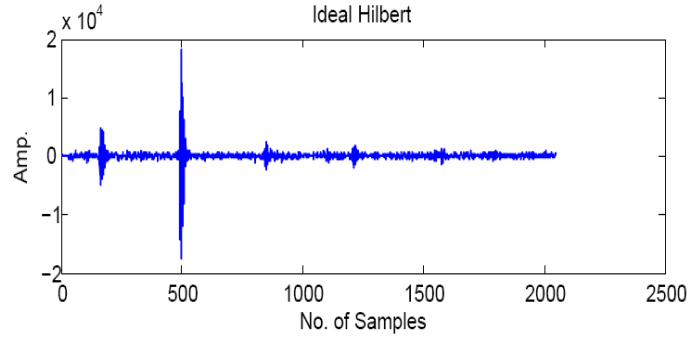

(b) 


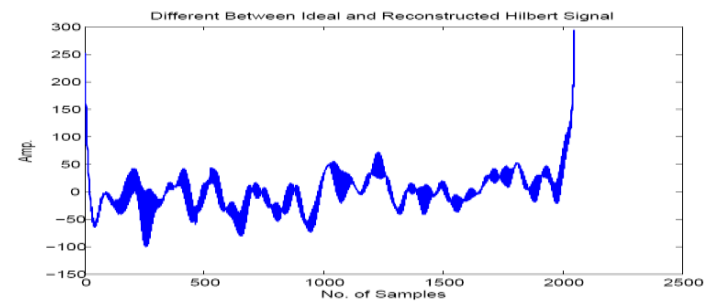

(c)

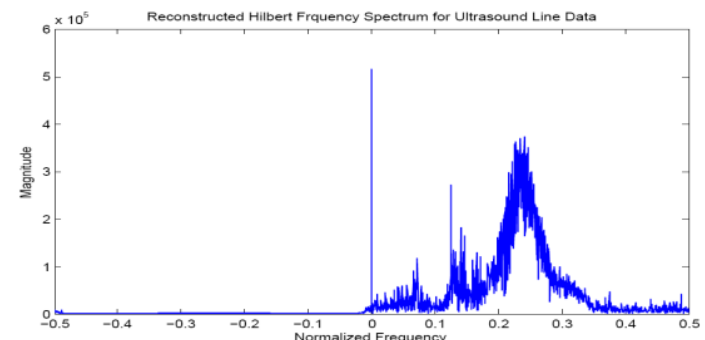

(d)

Fig.7: Hilbert filter apply to ultrasound line data. (a) Reconstructed FIR Hilbert, (b) Ideal Hilbert, (c) RMSE between reconstructed FIR and ideal Hilbert, (d) frequency spectrum of reconstructed FIR Hilbert.

\section{Envelope Detection}

The envelope of the in-phase and quadrature components was shown in Fig.8. Fig.8(a) mentioned the envelope of 60 samples of the ultrasound line data after delay and sum take after apply the 64-tap FIR Hilbert filter compare to the envelope which take after apply ideal Hilbert transform filter. The RMSE between the two envelopes equal to 0.2721 that means the two envelopes are closed to other. Fig.8(b) described the envelope take after apply the 64-tap FIR Hilbert filter for all the ultrasound line data compare to ideal Hilbert.

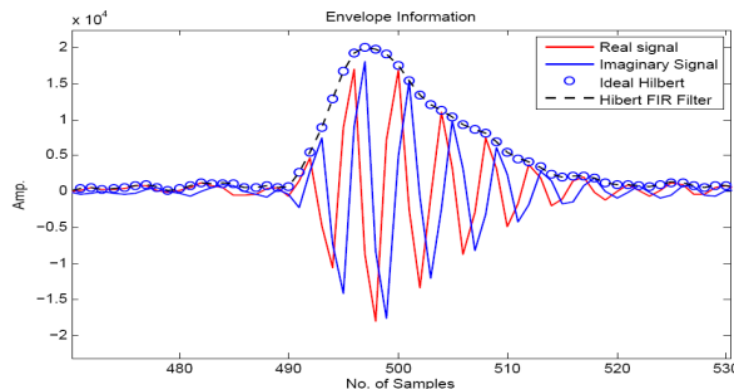

(a)

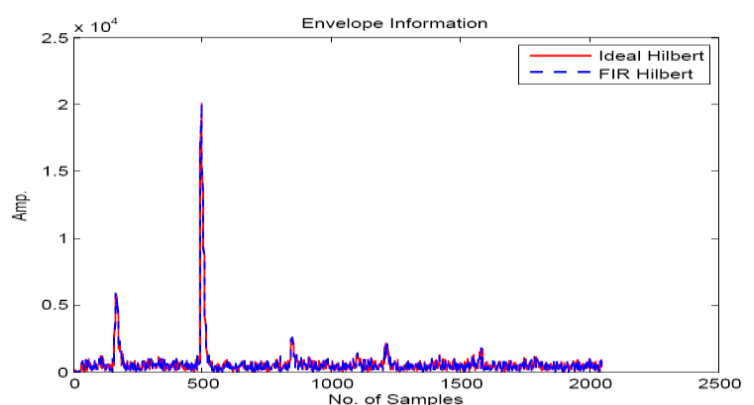

(b)

Fig.8: Envelope Detection. (a) The envelope of 60 samples of the in-phase and quadrature components after apply the 64-tap FIR Hilbert filter compare to the envelope take after apply ideal Hilbert transform filter, (b) The envelope take after apply the 64-tap FIR Hilbert filter for all the ultrasound line data compare to ideal Hilbert.

\section{Implementation Steps}

Fig.9(a) shown the analytical signal of 90 samples after implementation, Fig. 9(b) described the frequency spectrum of the implemented Hilbert filter to the ultrasound data line after beamforming. As can be shown the negative frequency was eliminated compare to the ideal filter frequency spectrum in Fig. 6(b). Fig. 9(c) show the implemented envelope take after apply the 64-tap implemented FIR Hilbert. The result was good if it compared to the simulation results in Fig.8. From the implementation the 64-tap filter uses only 32 multiplications. This was reducing the computational time by a half. Table 6 and 7 shown the 2x system clock (Discrete Pulse Generator) and continuous source (Discrete Pulse Generator) for the implementation system. The total estimated power consumption equal $0.8142 \mathrm{~W}$ and table 8 described the summary of the power consumption in the implementation. Table 9 shown the device utilization summary for the whole implementation, the used devices, available in the port, and the utilization in percentage. From the implementation result the power consumption and device utilization was acceptable. It is possible for the system to accept anther devices for further processing.
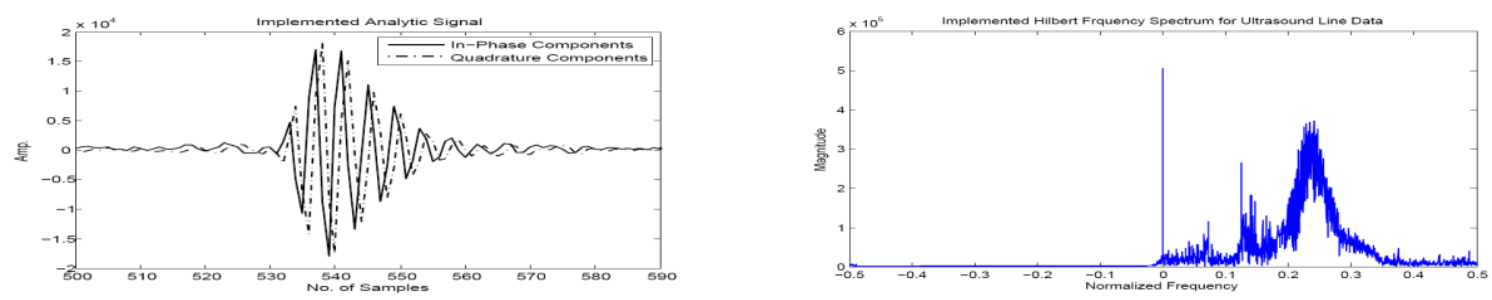
(a)

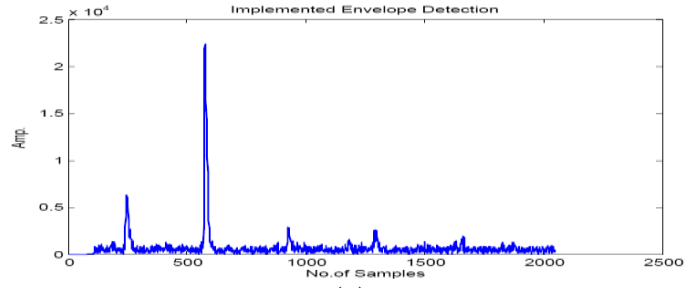

(c) (b)

Fig.9: Implementation. (a) The analytical signal, (b) Frequency spectrum after apply implemented FIR Hilbert filter, (c) The envelope detection.

Fig.10 had shown the implemented of the linear phase array image reconstruction to reconstruct image of six pins phantom from the data set, and the point spread function (PSF) of the first pin as indicated by white arrow in Fig.10(b) and Fig.10(d). Fig.10(a) described the image reconstruction after delay and sum and Fig.10(c) shown the image reconstructed after applying FIR Hilbert filter and envelope detection. As can be shown Fig. 10(c) provided best field of view and lateral resolution than Fig.10(a), because the envelope provided more signal strength. The PSF presented a quantitative measure of the beamforming quality.

Table 6: 2x System Clock (Discrete Pulse Generator)

\begin{tabular}{|l|l|}
\hline Parameter & Value \\
\hline Pulse type & Sample based \\
\hline Time (t) & Use simulation time \\
\hline Amplitude & 1 \\
\hline Period (secs) & 2 \\
\hline Pulse Width (\% of period) & 1 \\
\hline Phase delay (secs) & 0 \\
\hline Sample time & simulink_period \\
\hline $\begin{array}{l}\text { Interpret vector parameters } \\
\text { as 1-D }\end{array}$ & on \\
\hline
\end{tabular}

Table 7: Continuous Source (Discrete Pulse Generator)

\begin{tabular}{|l|l|}
\hline Parameter & Value \\
\hline Pulse type & Time based \\
\hline Time (t) & Use simulation time \\
\hline Amplitude & 1 \\
\hline Period (secs) & $2 *$ simulink_period \\
\hline Pulse Width (\% of period) & 50 \\
\hline Phase delay (secs) & 0 \\
\hline Sample time & 1 \\
\hline $\begin{array}{l}\text { Interpret vector parameters as } \\
\text { 1-D }\end{array}$ & on \\
\hline
\end{tabular}

Table 8: Power Consumption

\begin{tabular}{|l|l|l|l|l|l|}
\hline Power summary & $\mathbf{I}(\mathbf{m A})$ & $\mathbf{P}(\mathbf{m W})$ & Power summary & $\mathbf{I}(\mathbf{m A})$ & $\mathbf{P}(\mathbf{m W})$ \\
\hline Total Vccint 1.00V & 600.43 & 600.43 & Logic & - & 9.48 \\
\hline Total Vccaux 2.50V & 74.49 & 186.23 & Signals & - & 14.95 \\
\hline Total Vcco25 2.50V & 11.02 & 27.55 & Quiescent Vccint 1.00V & 522.01 & 522.01 \\
\hline Clocks & - & 50.63 & Quiescent Vccaux 2.50V & 74.00 & 185.00 \\
\hline DSP & - & 4.22 & Quiescent Vcco25 2.50V & 2.00 & 5.00 \\
\hline IO & - & 24.31 & $\begin{array}{l}\text { Total estimated power } \\
\text { consumption }\end{array}$ & & 814.21 \\
\hline
\end{tabular}

\section{CONCLUSIONS}

In this paper, embedded DSP for digital ultrasound imaging on FPGA is presented. The DSP was composed of FIR Hilbert transform filter, which was used to generate Quadrature component (Q) from the In-phase component (I) of the ultrasound data. The envelope (magnitude) of the received echo was computed. The implementation has been done in the Virtex-5 FPGA. The Hilbert filter is implemented in the form whereby the zero tap coefficients are not computed and therefore an order L filter uses only L/2 multiplications. This was reducing the computational time by a half. The power consumption and device utilization was acceptable. It is possible for the system to accept anther devices for further processing. The hardware architecture of the design provided flexibility. The system has the potential to lower the cost and speed up the development, thus offering new opportunities for more cost-effective systems. 


\section{$28^{\text {th }}$ NATIONAL RADIO SCIENCE CONFERENCE (NRSC 2011)}

April 26-28, 2011, National Telecommunication Institute, Egypt

Table 9: Device Utilization Summary

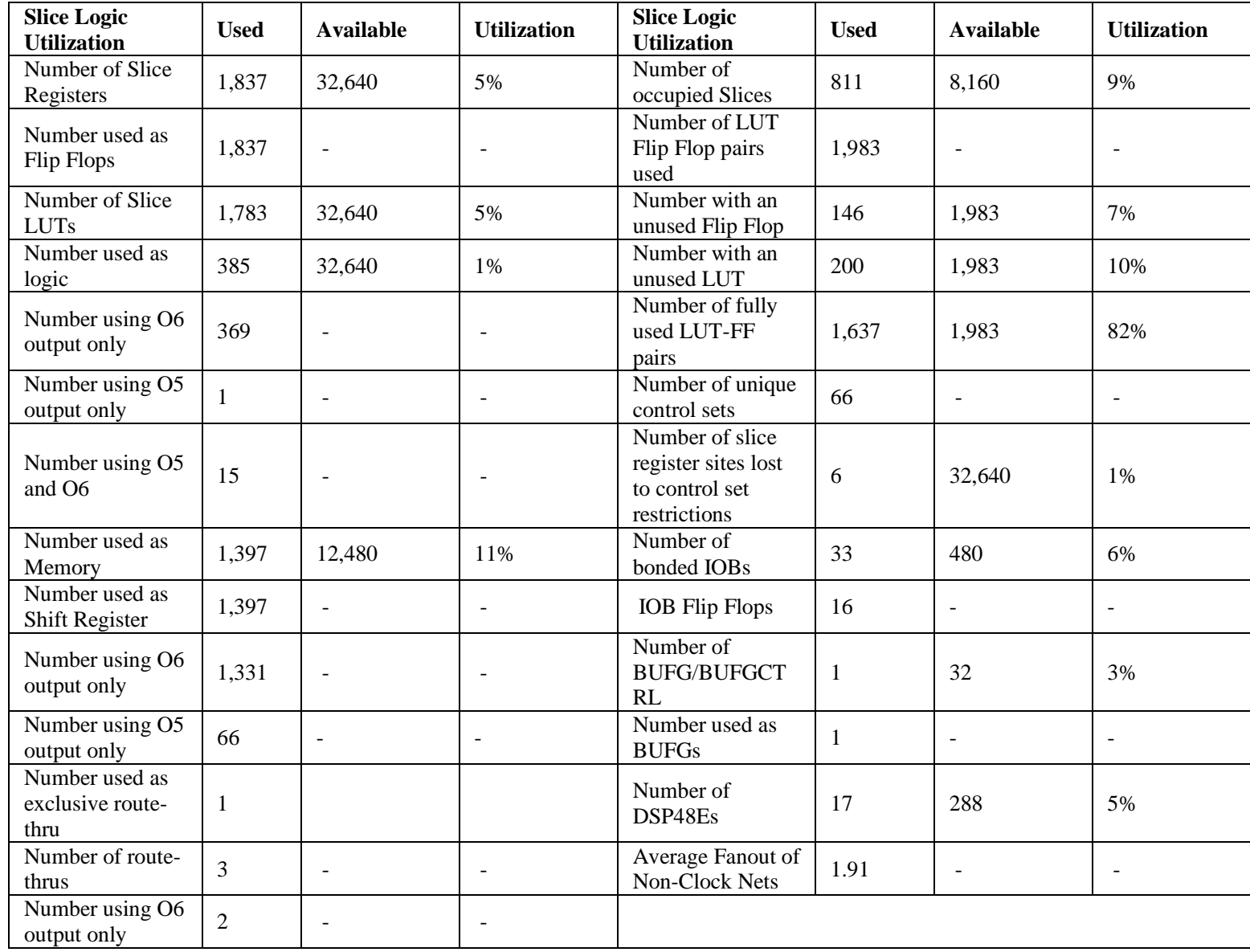

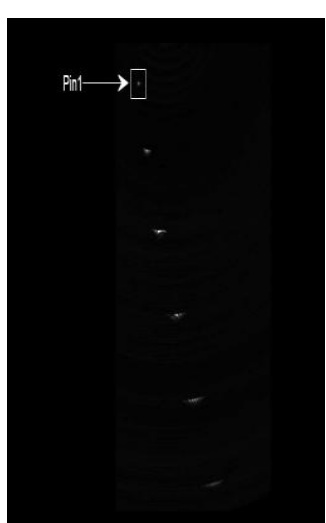

(a)

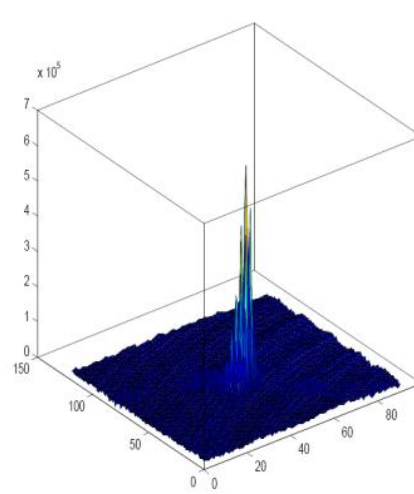

(b)

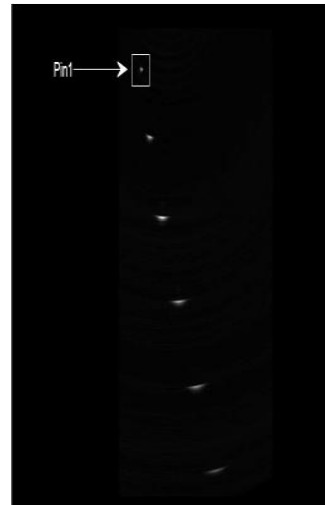

(c)

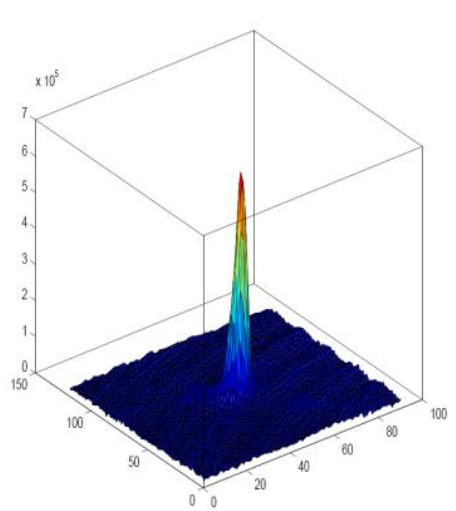

(d)

Fig.10: Image reconstruction and the PSF of the first pin. (a) Image after delay and sum, (b) PSF of the first pin in (a), (c) Image after applying FIR Hilbert filter and envelope detection, (d) PSF of the first pin in (c).

\section{REFERENCES}

[1] D. A .Christensen, Ultrasonic Bioinstrumentation, Jonh Wiley \& Sons, New York, 1988.

[2] J. A Zagzebski , Essentials of ultrasound physics, St Louis, Mo: Mosby, 1996.

[3] W. N.McDicken, Diagnostic Ultrasonics: Principals and Use of Instruments, JohnWiley \& Sons, 1976.

[4] S. Hughes, "Medical ultrasound imaging," Electronic Journals of Physics Education, vol. 36, no. 6, pp. 468-475, 2001.

[5] J. Souquet, "State of the art in digital broadband medical ultrasound imaging," C.R.Acad.Sci.Paris, Optical and Acoustic Imaging of Biological Media, pp. 1091-1098, 2001. 
[6] R. Reeder, C. Petersen, "The AD9271-A Revolutionary Solution for Portable Ultrasound," Analog Dialogue 41-07,Analog Devices, July 2007.

[7] J. O. Smith, Mathematics of the Discrete Fourier Transform (DFT), Center for Computer Research in Music and Acoustics (CCRMA), Department of Music, Stanford University, Stanford, California, 2002.

[8] A. V. Oppenheim and R. W. Schafer, Discrete-Time Signal Processing. NJ: Prentice-Hall, Englewood Cliffs, 1989.

[9] B.G. Tomov and J.A. Jensen, "Compact FPGA-Based Beamformer Using Oversampled 1-bit A/D Converters," IEEE Transactions on Ultrasonics, Ferroelectrics and Frequency Control, vol. 52, no. 5, pp. 870-880, May 2005.

[10] R. C. Gonzalez, R. E. Woods, Digital Image Processing, Pearson Prentice Hall, Upper Saddle River, New Jersey, 2008.

[11] B.D. Steinberg, "Digital beamforming in ultrasound," IEEE Transactions on Ultrasonics, Ferroelectrics and Frequency Control, vol. 39, no. 6, pp.716-721, 1992.

[12] C. Fritsch, M. Parrilla, T. Sanchez, O. Martinez, "Beamforming with a reduced sampling rate," Ultrasonics, vol. 40, pp. 599-604, 2002.

[13] S. R. Freeman, M. K. Quick, M. A. Morin, R. C. Anderson, C. S. Desilets, T. E. Linnenbrink, and M. O'Donnell, "Delta-sigma oversampled ultrasound beamformer with dynamic delays," IEEE Trans. Ultrason., Ferroelect., Freq. Contr., vol. 46, pp. 320-332, 1999.

[14] M. Kozak and M. Karaman, "Digital phased array beamforming using single-bit delta-sigma conversion with non-uniform oversampling," IEEE Trans. Ultrason., Ferroelect., Freq. Contr., vol. 48, pp. 922-931, 2001.

[15] M. A. Hassan, A. M. Youssef, and Y. M. Kadah, "Modular FPGA-Based Digital Ultrasound Beamforming," in Proc. Middle East Conference on Biomedical Engineering, Sharjah, UAE, 2011 (In Reviewer).

[16] A. M. Hendy ,M. A. Hassan, R. Eldeeb, D. Kholy, A. Youssef and Y. M. Kadah, "PC-Based Modular Digital ultrasound Imaging system," in Proc. IEEE Ultrason. Symp., pp.1330-1333, Rome, Italy, September 2009.

[17] M. A. Hassan, A. S. Mohamed, A M. Youssef, and Y. M. Kadah, "Signal Processing Methodologies for Digital Beamforming and Image Reconstruction in Ultrasound Imaging," in Proc. 27th National Radio Science Conference, Menouf, Egypt, March 2010.

[18] S. Sukittanon, S. G. Dame, "FIR Filtering in PSoC'TM with Application to Fast Hilbert Transform," Cypress Semiconductor Corp., Cypress Perform, November 2005.

[19] P. Montuschi and M. Mezzalama, "Survey of square rooting algorithms," Proc. IEEE, vol. 137, no. 1, pp. 31-40, 1990.

[20] J. Prado and R. Alcantara, “A fast square-rooting algorithm using a digital signal processor," Proc. IEEE, vol. 75, no. 2, pp. 262-264, 1987.

[21] T. K. Song and S. B. Park, "A new digital phased array system for dynamic focusing and steering with reduced sampling rate,” Ultrason. Imag., vol. 12, pp. 1-16, 1990.

[22] J. H. Chang, J. T. Yen, K. K Shung, “A Novel Envelope Detector for High-Frame Rate, High-Frequency Ultrasound Imaging," IEEE Transactions on Ultrasonics, Ferroelectrics and Frequency Control, vol. 54, no. 9, pp. 1792-1801, September 2007.

[23] M. O’Donnell and S.W. Flax, "Phase-aberration correction using signals from point reflectors and diffuse scatterers: measurements," IEEE Trans. Ultrason., Ferroelect., and Freq. Contr. 35, no. 6, pp. 768-774, 1988. 